\title{
Cesarean scar endometrioma: A rare case report with literature review
}

\begin{abstract}
Endometriosis is presence of endometrial glands and stroma outside uterine cavity. Scar endometriosis is an uncommon variant of extra pelvic endometriosis developing at scar site of previous abdominopelvic surgery. Pre-operative Diagnosis of scar endometriosis is often delayed due to its clinical similarity with other surgical conditions. We present a case of Pfannenstiel scar endometriosis diagnosed 6 years after cesarean section delivery and highlight pathogenesis, clinical and imaging features and various treatment options with an aim to increase awareness about this rare entity.
\end{abstract}

Keywords: endometriosis, scar endometrioma, extra pelvic endometriosis, casearean section
Volume 9 Issue 4 - 2019

\author{
Hemant Kumar Sharma,' Shivani Prashar ${ }^{2}$ \\ 'Pathologist, Government Hospital, India \\ ${ }^{2}$ Medical Officer, Government Hospital, India
}

Correspondence: Hemant Sharma, Department of Pathology, Government Hospital, Danapur Cantt, H.No. 167/I,MAP Quarters, India, 801503,Tel 8968865923, Email hemant|303@gmail.com

Received: June 13, 2019| Published: August 07, 2019
Abbreviations: SE, Scar endometrisois; LSCS, lower segment cesarean section

\section{Introduction}

Endometriosis, first described by German pathologist Carl von Rokitansky as cystosarcoma adenoids uterinum, is defined as presence of endometrial glands and stroma outside uterine cavity with a prevalence of $5-10 \%$ in women during reproductive age. ${ }^{1,2}$ Pelvic endometriosis is more common and seen in Uterine ligaments, Fallopian tubes, Pouch of Douglas, Ovaries and Pelvic peritoneum. On the contrary, extra pelvic endometriosis is rare entity with frequent localization in abdominal wall(most common), bladder, kidney, bowel, omentum, lymph nodes, lungs, pleura, extremities, umbilicus and hernia sacs sites. ${ }^{3}$ Scar endometrisois (SE), an extremely rare site (incidence $0.03-3.5 \%$ ) of extra pelvic endometriosis, is presence of endometriosis at or near previous surgery scar site as a painful discreet tumoral mass known as Endometrioma or admixed with native tissue. ${ }^{2,4}$ SE usually develops after General surgery and Obstetrics and Gynecological surgeries ,most commonly after hysterectomy (1.08-2\% cases) cesarean delivery $(0.03-0.04 \%$ cases $)$ and rarely after Appendicitis, Tubal ligation, Ectopic pregnancy, Inguinal herniorrhapy, in laparoscopic trocar tract and needle tract after diagnostic amniocentesis and in perineal episiotomy incision. ${ }^{1}$ However, few cases of Endometriosis of abdominal wall have been reported without any association with previous surgery. ${ }^{5}$ Most of the cases of SE are referred to Surgeons because its clinical similarity to incisional and inguinal hernias, abdominal wall tumor, Infective granuloma , lipoma, rectus sheath hematoma, keloid and other miscellaneous anterior abdominal wall masses and can cause unnecessary surgical interventions, inadvertent delayed or misdiagnosis and emotional and physical distress to the patient. ${ }^{4} \mathrm{We}$ are reporting a rare case of scar endometriosis diagnosed 6 years after endometrioma developed in lower segment cesarean section (LSCS) scar to increase awareness about this benign treatable entity for correct timely diagnosis.

\section{Case details}

28 years old P2L2female patient reported to Gynecology department with a painful swelling at left angle of previous LSCS pfannenstiel scar for 6 years. She delivered her first Full term Alive and Healthy baby by LSCS 6 years back. She first felt pain at operated site 3 months after the surgery. It was cyclic and used to subside without any treatment. About 3 years back, she again conceived and delivered Full term, Alive and Healthy baby by LSCS. Now she presents with a tender swelling measuring $4 \times 3 \times 2 \mathrm{~cm}$ at left angle of healed pfannenstiel scar. It was well circumscribed, firm and situated in subcutaneous plane. Rest of her systemic examination, vital parameters, laboratory investigations and sonographic examination were unremarkable without any evidence of pelvic endometriosios (Figure 1).

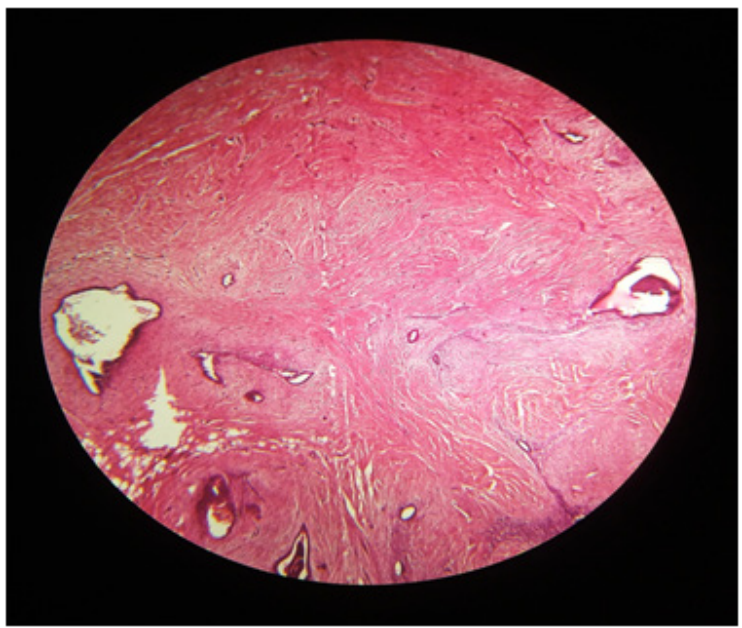

Figure I Photomicrograph showing benign endometrial glands and stroma surrounded by fibrous scar tissue consistent with Endometriosis (Hematoxylin and eosin $\times 100$ ).

Ultrasonogram (USG) of swelling showed well defined lesion measuring 2.6x1.5.x.15cm in left pelvic region at the pfannenstiel scar site which was predominantly hypoechoic with areas of hyeprechogenecity within. Based on USG findings along with clinical history, a diagnosis of Scar endomerisois was made. Patient was taken up for wide local excision of swelling with surrounding margins of 1 $\mathrm{cm}$. Gross examination of excised tissue revealed irregular, grayish black fibro fatty mass with areas of congestion and hemorrhage. 
Histopathology examination revealed endometrial glands surrounded by endometrial stroma, embedded in fibro collagenous tissue and hemosiderin pigment at places without any evidence of malignancy (Figure 2). All histopathology findings were consistent with Scar endometriosis. On follow up period for 6 months, she did not report for any recurrence of swelling and was completely relived of her cyclic symptoms.

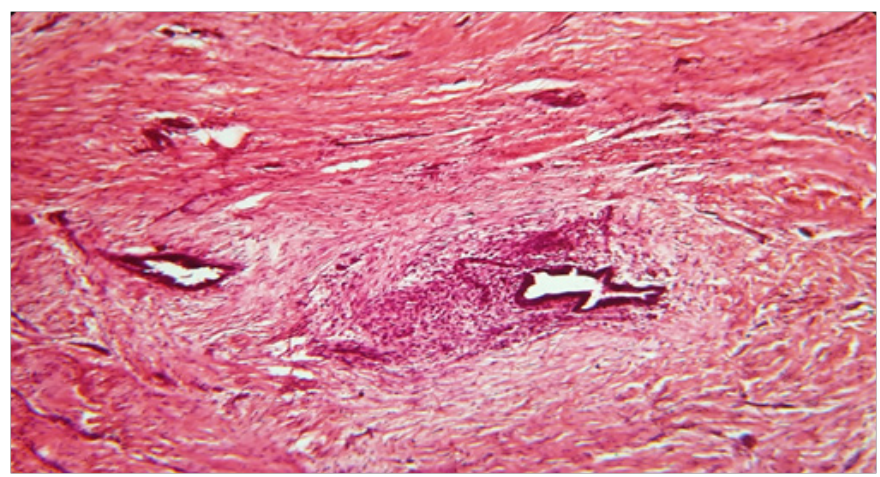

Figure 2 Photomicrograph showing benign endometrial glands and stroma surrounded by fibrous scar tissue consistent with Endometriosis (Hematoxylin and eosin $\times 200)$.

\section{Discussion}

Various theories like Metaplasia theory suggesting differentiation of primitive pluripotent mesenchymal cells in to endometrial tissues at sites other then uterine cavity and Transport theory suggesting transportation of endometrial cells to extra uterine sites have been postulated about development of endometriosis. ${ }^{1,6}$ Another theory postulates ability of endometrial cells to regulate cytotoxic immune activity by diminishing natural killercell activity in clearing endometrial cells and their capability to resist immune-mediated apoptosis enabling survival of ectopic endometrial cells in peritoneum. ${ }^{3,7}$ As proliferating capacity of end differentiated endometrial cells is limited, Most Plausible hypothesis favoring development of scar site Endometrioma appears to be transportation and inadvertent inoculation of endometrial cells during surgical procedures followed by cyclical hormonal stimulation by estrogen similar to normal endometrium. Endometrioma usually develops 3 months to 10years (average 3.6years) after the surgeries but time between surgery and diagnosis varies. $^{2}$ Our patient underwent LSCS 6 years ago. Although rare entity, Endometrioma develops more commonly post-hysterotomy because of increased cellular replication and regenerative capacity of early decidua due to higher capability for pluripotency. ${ }^{1}$ As in our case, Pfannenstiel incision post LSCS is the most common site for development of SE possibly because wider dissection of tissue planes compared to vertical midline incisions. Usually patients presents with symptoms of severe dysmenorrhea, dyspareunia, menstrual irregularities and infertility. Clinically, endomtrioma presents as discoloured tender swelling responding in cyclic hormonal changes located in a surgical scar area. ${ }^{4}$ Thorough clinical and sonographic evaluation of all SE patients is necessary to rule out concomitant pelvic endomteriosis seen $24 \%$ of all SE patients. ${ }^{1,3}$ Our patient did not show any evidence of pelvic endometriosis. Due to variable presentation, lack of awareness and clinical similarity to other lesions specific to scar site, preoperative diagnosis of scar endometrioma is very difficult leading to unnecessary delays in correct diagnosis and unwarranted surgeries. In fact, most of the cases of scar endometrioma are diagnosed post-operatively during histopathological examination. Various imaging diagnostic modalities like USG, CT(Computed tomography) and Magnetic resonance (MR) imaging have been used for establishing pre-operative diagnosis of scar endometriosis depending on the phase of the patient's menstrual cycle, the chronicity of the process, the number of stromal and glandular elements, and the amount of bleeding and associated inflammation. ${ }^{6}$ USG shows non-specific findings of solid, hypoechoic and vascularized mass with speculated margins infiltrating the surrounding tissue with scattered internal echoes representing fibrotic strands. Francicsa et al. made accurate pre-operative diagnosis in 12 patients by using USG with Colour Doppler findings along with clinical examination. ${ }^{8}$ However, abdominal wall endometriosis can easily be missed in obese patients if appropriate USG probe is not used. ${ }^{5}$ CT scan of swelling shows a solid, well-circumscribed soft tissue mass directly associated with an area of surgical scarring while MR Imaging shows a hyper intense heterogeneous nodule associated with anterior abdominal or pelvic wall surgical scarring. MR Imaging is more useful in young patients due to its improved tissue characterization and lack of ionizing radiation. Also, MR imaging is superior compared to CT scan particularly in patients who are symptomatic or have extensive lesions with deep infiltration by depicting the delineation between muscles and abdominal subcutaneous tissues and infiltration of abdominal and pelvic wall structures, to identify hemorrhage associated with endometriotic lesions and to accurately and safely plan surgical resection and can be more helpful when diagnosing small lesions due to its high spatial resolution due to its ability to detect detecting the planes between muscles and abdominal subcutaneous tissue., ${ }^{2,4,6}$ Recently various studies like Veda et al stressed on the importance of FNAC in early diagnosis of SE and excluding other mimicking entities like metastatic deposit, desmoid tumor, lipomas, cysts, fat necrosis ,hematoma or abscess. FNAC of SE shows sheets of epithelial cells, spindled stromal cells and variable number of hemosiderin laden macrophages and confirmation of diagnosis requires presence of any two out of there features. ${ }^{9}$ FNAC, CT and MRI were not done in our patient to save cost and time as she was feeling severe pain and above all, these investigations would not have contributed much with working clinical diagnosis of scar endometriosis and choice of treatment of wide local excision in all suspected cases of scar endometriosis. Although Various Medical and Surgical treatment modalities have been tried, treatment of choice remains wide excision of the lesion with/without mesh placement depending upon size of the lesion along with histologically proven, surgical-free margin of $1 \mathrm{~cm}$ to prevent recurrence. ${ }^{3}$ In symptomatic patients, Diagnostic laparoscopy can be combined with excision to assess pelvic localization and exclude intraperitoneal spread. Medical treatment used for relief of symptoms includes Progestogens, Oral contraceptive pills, Danazol and Gonadotropin agonist like leuprolide acetate have yielded only partial relief without any reduction in size., ${ }^{2,3}$ Follow up and reexcision is required in recurrent cases(incidence $4.3 \%$ after surgery) with thorough evaluation to rule out any malignancy. ${ }^{3}$ Malignant transformation of endometriosis is a rare(seen in $0.3-1 \%$ of cases), rapidly progressive complication with 20 -month survival rate is only $57 \%$ and can be attributed to combination of oxidative stress from recurrent hemorrhage, inflammation, and hyperestrinism., Till 2017, 22 cases of carcinoma arising in scar endometroisois reported, out of which clear cell carcinoma was approximately $(77.3 \%, 17 / 22)$ followed by endometrioid carcinoma $(13.6 \%, 3 / 22)$ and serous carcinoma $(0.09 \%, 2 / 22){ }^{10}$ If there is frequent recurrence or endometrioma is fast-growing, large and ulcerating, malignancy should be suspected. Incidence of scar endometriosis can be reduced by thorough cleaning and irrigation of surgical wound with high jet solution before closure in all abdominopelvic procedures. ${ }^{2,7}$ 


\section{Conclusion}

Scar endomteriosis should be suspected in any women in reproductive age group presenting with a painful swelling in the abdominal scar especially with a history of previous gynecological or obstetrical surgery. Clinical similarity with various surgical lesions of anterior abdominal wall and vague symptoms often delay diagnosis and casue physical and emotional stress to the patient. Imaging modalities like MRI and FNAC should be used for accurate pre-operative diagnosis. Treatment of choice is wide local excision with $1 \mathrm{~cm}$ margin with regular follow-up to detect recurrence. Stress must be laid on thorough cleaning and washing of surgical wound all abdominopelvic surgeries to avoid iatrogenic implantation of endometrium.

\section{Acknowledgements}

None.

\section{Funding}

None.

\section{Conflicts of interest}

The authors declare that there is no conflict of interest.

\section{References}

1. Chatterjee SK. Scar endometriosis: A clinicopathological study of 17 cases. Obstet Gynecol. 1980;56(1):81-84.
2. Gupta P, Gupta S. Scar Endometriosis: a case report with Literature review. Acta Medica Iranica. 2015;53(12):793-795.

3. Mistrangelo M, Gilbo N, Cassoni P, et al. Surgical scar endometriosis. Surg Today. 2014;44(4):767-772.

4. Leite GK, Carvalho LF, Korkes H, et al. Scar endometrioma following obstetric surgical incisions: retrospective study on 33 cases and review of the literature. Sao Paulo Med J. 2009;127(5):270-277.

5. Tomás E, Martín A, Garfia C, et al. Abdominal wall endometriosis in absence of previous surgery. J Ultrasound Med. 1999;18(5):373-374.

6. Gidwaney R, Badler RL, Yam BL, et al. Endometriosis of abdominal and pelvic wall scars: multimodality imaging findings, pathologic correlation, and radiologic mimics. Radiographics. 2012;32(7):2031-2043.

7. Ding DC, Hsu S. Scar endometriosis at the site of cesarean section. Taiwan J Obstet Gynecol. 2006;459(3):247-249.

8. Francica G, Giardiello C, Angelone G, et al. Abdominal wall endometriomas near cesarean delivery scars: sonographic and color doppler findings in a series of 12 patients. J Ultrasound Med. 2000;22(10):1041-1047.

9. Veda P, Srinivasaiah M. Incisional endometriosis: diagnosis by fine needle aspiration cytology. $J$ Lab Physicians. 2010;2(2):117-120.

10. Wei CJ, Huang SH. Clear cell carcinoma arising from scar endometriosis: A case report and literature review. Tzu Chi Med J. 2017;29(1):55-58. 